\title{
ASPECTOS ANATÓMICOS Y BIOMÉTRICOS DE LA INERVACIÓN DEL MÚSCULO CORACOBRAQUIAL Y SUS PUNTOS MOTORES
}

\author{
ANATOMICAL AND BIOMETRICAL ASPECTS OF THE \\ CORACOBRACHIALIS MUSCLE INNERVATION AND THEIR MOTOR POINTS
}

\author{
Enrique Olave
}

\begin{abstract}
OLAVE, E. Aspectos anatómicos y biométricos de la inervación del músculo coracobraquial y sus puntos motores. Int. J. Morphol., 22(4):323-326, 2004.

RESUMEN: El músculo coracobraquial está inervado por uno o dos ramos que le envía el nervio musculocutáneo. Con el propósito de obtener informaciones biométricas de la inervación de este músculo, estudiamos 42 miembros superiores, de 21 cadáveres de individuos adultos, brasileños, fijados en formol al 10\%. Se realizó cuidadosa disección, poniendo énfasis en el nervio musculocutáneo y los ramos de inervación para el músculo coracobraquial, registrando su origen respecto a una línea transversal extendida entre los dos epicóndilos humerales (LBE), longitud, relación músculo-nervio respecto a LBE, subdivisión de sus ramos y localización de sus puntos motores en relación a esta línea. Encontramos sólo un ramo de inervación para este músculo, en 11 casos del lado izquierdo (52,4\%) y 6 casos del lado derecho (28,6\%); con dos ramos hubo 7 casos del lado izquierdo (33,3\%) y 11 del lado derecho $(52,4 \%)$; con tres ramos observamos 3 casos del lado izquierdo (14,3\%) y 4 del lado derecho (19\%). El nervio musculocutáneo, pasó a través de las fibras del músculo en 35 casos del total estudiado (83,3\%) y lo hizo a una distancia promedio de 227,9 mm (DS de 20,2) en el lado derecho y de 225,4 mm (DS de 40,6) en el izquierdo. El punto de origen del ramo muscular (R1) cuando las muestras presentaron sólo un ramo de inervación, fue en promedio de $266,3 \mathrm{~mm}$ (DS de 11,0) en el lado derecho y 252,5 mm (DS de 18,9) en el izquierdo. Para el punto de origen de R1, cuando las muestras presentaron dos ramos, registramos un promedio de 255,4 $\mathrm{mm}$ (DS de 15,2) en el lado derecho y de 265,9 mm (DS de 17,1) en el izquierdo. En este mismo grupo, el punto de origen de R2 se localizó a 239,2 mm en promedio (DS de 18,8) en el lado derecho y de $247,7 \mathrm{~mm}$ (DS de 19,3) en el izquierdo. Los datos presentados complementarán el conocimiento anatómico y podrán servir de soporte morfológico al área de electroestimulación y a los procedimientos quirúrgicos que involucren a estas estructuras.
\end{abstract}

PALABRAS CLAVE: 1. Anatomía; 2. Músculo coracobraquial; 3. Inervación.

\section{INTRODUCCIÓN}

El músculo coracobraquial se localiza en la región anterior del brazo, originándose junto con la cabeza corta del músculo bíceps braquial en el proceso coracoides, manteniendo una estrecha relación con el nervio musculocutáneo, responsable de la inervación de los músculos de esta región. Este último emite uno o más ramos motores para el músculo coracobraquial, los que penetran en su vientre muscular. Por otra parte, la mayoría de las descripciones anatómicas señalan que el nervio musculocutáneo pasa a través de las fibras del mismo (Sunderland, 1985; Williams et al.,1995; Snell, 2002; Moore \& Dalley, 2003). Sin embargo, no hay datos sobre el número de ramos, longitud de los mismos, su distribución y la localización de sus puntos motores.

El propósito de esta investigación fue conocer detalles biométricos de la inervación del músculo coracobraquial.

\section{MATERIAL Y MÉTODO}

Para este estudio fueron analizados 42 miembros superiores, derechos e izquierdos, de cadáveres de individuos adultos, brasileños, fijados en formol al $10 \%$. Utilizando una lupa Ranson se realizaron disecciones planificadas en cada miembro, registrando la relación entre el músculo coracobraquial y el nervio musculocutáneo, número de ramos emitidos hacia el músculo, nivel de origen de éstos, en relación a una línea trazada entre las partes más prominentes de los epicóndilos humerales - línea biepicondilar (LBE) - distancia entre el punto motor y la LBE, longitud de cada ramo. Posteriormente, los datos fueron analizados estadísticamente con el programa SPSS 11.0 obteniendo promedios, desviación standard, comparación entre ambos lados, correlación entre punto de origen y local del punto motor con la distancia acromionlínea biepicondilar. 


\section{RESULTADOS}

Con relación al número de ramos, encontramos sólo un ramo de inervación para el músculo en estudio, en 11 casos del lado izquierdo $(52,4 \%$ ) y 6 casos del lado derecho (28,6\%); con dos ramos hubo 7 casos del lado izquierdo $(33,3 \%)$ y 11 del lado derecho $(52,4 \%)$; con tres ramos observamos 3 casos del lado izquierdo (14,3\%) y 4 del lado derecho (19\%).

$\mathrm{Al}$ considerar el total de casos (42), con un ramo observamos $40,5 \%$ de las muestras; con dos ramos, $42,9 \%$ y con tres ramos, $16,6 \%$.

Con respecto al origen de los ramos musculares, éstos procedieron desde el nervio musculocutáneo, en 19 casos del lado izquierdo y 18 del lado derecho $(88,1 \%$ del total de casos). También se originaron desde el fascículo lateral del plexo braquial, en 2 casos del lado izquierdo y 3 del lado derecho (11,9\% del total).

En cuanto a la relación entre el músculo coracobraquial y el nervio musculocutáneo, éste pasó a través de sus fibras, en 35 casos del total estudiado (83,3\%), 18 en el lado derecho y 17 en el izquierdo. Sólo en 7 casos (16,7\%), 3 en el derecho y 4 en el izquierdo, no hizo su trayecto a través del vientre muscular.

El punto a través del cual el nervio musculocutáneo pasó, a través del músculo, fue registrado en relación a la LBE, siendo en promedio de 227,9 mm con una DS de 20,2 en el lado derecho y de $225,4 \mathrm{~mm}$ con una DS de 40,6 en el izquierdo. Al comparar ambos lados no se obtuvo una diferencia estadísticamente significativa.

El punto de origen del ramo muscular (R1) cuando las muestras presentaron sólo un ramo de inervación, se registró en relación a la LBE y fue en promedio de $266,3 \mathrm{~mm}$ con una DS de 11,0 en el lado derecho y $252,5 \mathrm{~mm}$ con una DS de 18,9 en el izquierdo. Comparando ambos lados, la diferencia encontrada no fue estadísticamente significativa.

Para el punto de origen de R1 cuando las muestras presentaron dos ramos, siempre en relación a LBE, registramos un promedio de $255,4 \mathrm{~mm}$ con una DS de 15,2 en el lado derecho y de $265,9 \mathrm{~mm}$ con una DS de 17,1 en el izquierdo. En la comparación de lados, las diferencias no fueron estadísticamente significativas.

Siempre en los casos que presentaron dos ramos de inervación, el punto de origen de $\mathrm{R} 2$ se localizó a un promedio de 239,2 mm con una DS de 18,8 en el lado derecho y de
247,7 mm con una DS de 19,3 en el izquierdo, siempre respecto a LBE.

En los casos que presentaron tres ramos, las distancia registradas se presentan en la Tabla I.

Tabla I. Promedios de las distancias entre el punto de origen de los ramos musculares y la línea biepicondilar (LBE), cuando encontramos tres ramos de inervación ( $\mathrm{mm})$.

\begin{tabular}{lcc}
\hline RAMOS & L.D. & L.I. \\
R1 & $261+18,5$ & $247,7+4,5$ \\
R2 & $251,7+19,8$ & $237,0+8,1$ \\
R3 & $232,0+38,1$ & $226,3+8,5$ \\
\hline
\end{tabular}

Con relación a los puntos motores, el R1 ingresó en el vientre muscular a una distancia promedio con respecto a LBE, de $259 \mathrm{~mm}$ (DS de 23,7) en el lado derecho y de 245,2 mm (DS de 18,9) en el izquierdo. Al comparar los lados, las diferencias no fueron estadísticamente significativas.

En el grupo con dos ramos, el R1 entró en el vientre muscular a una distancia promedio de $247,6 \mathrm{~mm}$ (SD de 12,6 ) en el lado derecho y de $257,6 \mathrm{~mm}$ (SD de 12,3). Al comparar los lados, no hubo diferencias estadísticamente significativas.

También realizamos una comparación entre los puntos de ingreso de $\mathrm{R} 1$ en el grupo con un ramo de inervación y de $\mathrm{R} 1$ con dos ramos de inervación en ambos lados, no encontrando diferencias estadísticamente significativas.

En el grupo con dos ramos, el R2 ingresó en el vientre muscular a una distancia promedio con respecto a LBE de 232,7 mm (SD de 21,5) en el lado derecho y de 244,3 $\mathrm{mm}$ (DS de 16,3) en el izquierdo. Al comparar ambos lados, las diferencias encontradas no fueron estadísticamente significativas.

Las distancias registradas entre los puntos motores y la LBE en los casos con tres ramos son mostradas en la Tabla II.

Tabla II. Distancias promedios entre el punto motor y la LBE en el grupo de casos con tres ramos.

\begin{tabular}{lcc}
\hline Ramos & Lado derecho & Lado izquierdo \\
R1 & $254,3+21,2$ & $241,7+6,6$ \\
R2 & $246,7+23,5$ & $232,7+5,8$ \\
R3 & $218,7+46$ & $226,0+9,2$ \\
\hline
\end{tabular}

Con respecto a la longitud de los ramos, el R1 del grupo de casos que tenían sólo un ramo de inervación para el músculo en cuestión, midió en promedio 18,3 mm (DS de 7,5 ) en el lado derecho y 22,2 mm (DS de 17,6) en el izquierdo. Al comparar ambos lados, no hubo diferencias estadísticamente significativas. 
En el grupo con dos ramos de inervación, el R1 tuvo una longitud promedio de 17,1 mm (DS de 11,1) en el lado derecho y de $18,7 \mathrm{~mm}$ (DS de 10,6) en el izquierdo. No hubo diferencias estadísticamente significativas al comparar los lados derecho e izquierdo.

Comparando R1 del grupo con un ramo y R1 del grupo con dos ramos de inervación, en ambos lados, no hubo diferencias estadísticamente significativas.

La longitud de R2 en el grupo con dos ramos de inervación fue de 12,9 mm (DS de 9,4) en el lado derecho y de 11,4 mm (DS de 9,1) en el izquierdo. Al comparar ambos lados, las diferencias encontradas no fueron significativas.

\section{DISCUSIÓN}

La inervación del músculo coracobraquial proviene del nervio musculocutáneo, el cual es ramo terminal del plexo braquial y está destinado a los músculos de la región anterior del brazo, otorgando también inervación sensitiva a la región lateral del antebrazo (Testut \& Latarjet, 1971; Chusid, 1974; Williams et al.,1995; Moore \& Dalley, 2002; Snell, 2002).

Los clásicos textos de Anatomía describen que el músculo coracobraquial recibe un ramo de inervación, el que se desprende del nervio musculocutáneo, disposición que encontramos en el 40,5\% de nuestra serie. Son pocos los autores (Testut \& Latarjet; Lazorthes, 1955) que describen la existencia de dos ramos de inervación, situación que observamos en el 42,9\% de los casos, aún más, con tres ramos hubo un $16,6 \%$, número no registrado en la literatura consultada. Testut \& Latarjet refieren que los dos ramos mencionado por ellos, frecuentemente se originan a partir de un ramo común $(53,5 \%)$, hecho no registrado en esta pesquisa.

El origen del nervio está muy claro en la literatura (Testut \& Latarjet; Sunderland; Tountas \& Bergman (1993); Williams et al.; Moore \& Dalley), en la mayoría de los casos, a partir del nervio musculocutáneo y, a veces, procedente del fascículo lateral del plexo braquial, disposición con la que concordamos, ya que esto último lo encontramos sólo en un $11,9 \%$ del grupo estudiado.

Respecto a la relación de trayecto entre el nervio musculocutáneo y el músculo en cuestión, autores como Goss (1976); Chusid; Gardner et al.(1977); Hamilton (1977); Sunderland; Moore \& Dalley; Snell) han señalado que el ner- vio pasa a través de las fibras del músculo coracobraquial. Concordamos con esta relación, la que se da en la mayoría de los casos, pero no podemos dejar de mencionar que, en aproximadamente un sexto de los individuos, no existe el trayecto a través de las fibras del músculo, validando lo descrito por Tountas \& Bergman.

Datos biométricos del punto en que el músculo es perforado por el nervio musculocutáneo, no encontramos en la literatura, por lo que su conocimiento se hace importante en las diferentes acciones que se realizan respecto al mismo, por ejemplo, cuando se debe anestesiar este nervio, así este aporte puede dar una mejor visión del área a inyectar.

Registros de las distancias de origen, número, longitud de los ramos musculares y su distribución son escasos, por lo tanto los resultados presentados complementarán el conocimiento anatómico de la inervación del músculo en estudio.

Se sabe que conociendo el o los puntos motores en un determinado músculo, podemos estimularlo eléctricamente para mejorar su condición en caso de músculos atróficos, sobretodo en los de mayor tamaño, ya que a veces el nervio principal se divide en varios ramos secundarios que ingresan en el vientre, distanciados uno de otro (Olave \& Braga, 2003). Ya fue demostrado por Liu et al. (1996) que al estimular uno de los puntos motores en la cabeza larga del tríceps braquial de conejos, existía diferencia en el grado de extensión del codo, siendo mayor cuando se estimulaba el punto distal (el otro punto es proximal) y que, cuando se estimularon ambos, hubo un mayor rango de extensión que cuando se hizo individualmente.

El conocimiento de la localización de estos puntos puede tener una importante aplicación funcional, ya que la estimulación eléctrica de múltiples puntos motores producirá una óptima acción muscular.

En los grupos con uno, dos y tres ramos de inervación, los ramos principales pueden subdividirse o no, pudiendo ingresar R1 como un ramo único en dos tercios de los casos, subdividiéndose en dos ramos secundarios separados por una pequeña distancia en menos de un tercio de las muestras y subdividiéndose en tres ramos menores en un solo caso. Los ramos R2 y R3 ingresan en el músculo como ramo único en la mayor parte de los casos, salvo excepciones en que pueden ingresar subdivididos en dos ramos secundarios.

Las informaciones biométricas de la inervación del músculo coracobraquial obtenidas en esta investigación, sin duda, complementarán los conocimientos ya descritos y serán un aporte a las áreas de electroestimulación y anatomía quirúrgica de la región. 
OLAVE, E. Anatomical and biometrical aspects of the coracobrachialis muscle innervation and their motor points. Int. J. Morphol., 22(4):323-326, 2004.

SUMMARY: The coracobrachialis muscle is innervated by one or two branches sent by the musculocutaneous nerve. Under the purpose to obtain some biometric information on their innervation, we studied 42 upper extremities from 21 cadavers formolized of adult individuals. A carefull dissection of the musculocutaneous nerve was performed and the coracobraquial muscle innervation branches were examined on basis of a transversal line extended between the two humeral epicondiles (BEL). Therefore the origins of the motor branches in relationship to BEL were recorded, also length, subdivisión of branches and localization of their motor points were taken in account respect to this line. In 11 cases we found only one innervation branch to the respective muscle on the left side (52.4\%) and 6 cases on the right side (28.6\%); with two branches we found 7 cases on the left side (33.3\%) and 11 cases on the right side (52.4\%); with three branches we found 3 cases on the left side (14.3\%) and 4 cases in the right side (19\%). The musculocutaneous nerve went through the muscle fibers in 35 cases (83.3\%), from the total studied cases and made it to an average distance of $227.9 \mathrm{~mm}$ (SD of 20.2) on the right side and $225.4 \mathrm{~mm}$ (SD of 40.6) on the left side, respect to the BEL.

The source of origin for the muscular branch (R1) under only one innervation branch, had an average of 266.3 mm (SD of 11.0) on the right side and $252.5 \mathrm{~mm}$ (SD of 18.9) on the left side respect to the BEL. For the origin of R1 under two simple branches group, it was registrated an average of $255.4 \mathrm{~mm}$ (SD of 15.2) on the right side and $265.9 \mathrm{~mm}$ (SD of 17.1) on the left side. In the same group the place for the origin of R2 was localized at an average of $239.2 \mathrm{~mm}$ (SD of 18.8) on the right side and $247.7 \mathrm{~mm}$ (SD of 19.3) in the left side. These data presented here contributes to the anatomical knowledge of this zone and can be used as a morphological support to the electrostimulation area as well as surgical procedures involving such structures.

KEY WORDS: 1. Anatomy, 2. Coracobrachialis muscle; 3. Innervation.

\section{REFERENCIAS BIBLIOGRÁFICAS}

Chusid, J. Neuroanatomía correlativa y Neurología funcional. México, El Manual Moderno, 1974.

Gardner, E.; Gray, D. \& O'Rahilly, R. Anatomia. Estudio por regiones del cuerpo humano. 2. ed. México, Salvat, 1977.

Goss, Ch M. Gray Anatomía. 29. ed. Barcelona, Salvat, 1976.

Hamilton, W. J. Textbook of Human Anatomy. 2. ed. U.K., Macmillan, 1977.

Lazorthes, G. Sistema Nervioso Periférico. Descripción-Sistematización-Exploración. Barcelona, Toray-Masson, 1955.

Liu, J.; Lau, H.; Pereira, B.; Kumar, V. \& Pho, R. Terminal nerve branch entries (motor points) of forearm muscles: a comparative study between monkey and human. Acta Anat., 155:41-9, 1996.

Moore, K. \& Dalley, A. Anatomía con orientación clínica. 4. ed. Buenos Aires, Panamericana, 2002.

Olave, E. \& Braga, M.T.T. Inervación del músculo semitendinoso, biometría de sus ramos y localización de sus puntos motores. Int. J. Morphol. 21(2):161-5, 2003.

Snell, R. Anatomía Clínica para estudiantes de Medicina. 6. ed. México, McGraw Hill Interamericana, 2002.

Sunderland, S. Nervios periféricos y sus lesiones. 2. ed. Barcelona, Salvat, 1985.
Testut, L. \& Latarjet, A. Tratado de Anatomía Humana. 9a Ed. Barcelona, Salvat, 1969. V. I.

Tountas, Chris P. \& Bergman, R. Anatomic variations of the upper extremity. New York, Churchill Livingstone Inc., 1993.

Williams, P.; Warwick, R.;Dyson, M. \& Bannister, L. Gray Anatomia. 37 ed. Rio de Janeiro, Guanabara Koogan, 1995.

Dirección para correspondencia

Prof. Dr. Enrique Olave

Facultad de Medicina

Universidad de La Frontera

Casilla $54 \mathcal{D}$

Temuco - CHILE

Email:eolave@ufro.cl

Recibido : 10-07-2004

Aceptado: 14-10-2004 